\title{
Highly Regio-, Enantio-, and Exo-selective Diels-Alder Reactions Enabled by a Bispyrrolidine Diboronate
}

\author{
Yuan-He Li ${ }^{1}$, Su-Lei Zhang ${ }^{1}$, Bo Xiao ${ }^{2}$, Tian-Yu Sun ${ }^{3}$, Jia-Hua Chen ${ }^{1 *}$, Yun-Dong Wu ${ }^{1,2,3 *}$, \\ Zhen Yang $1,3,4 *$
}

${ }^{1}$ State Key Laboratory of Bioorganic Chemistry and Molecular Engineering of Ministry of Education and Beijing National Laboratory for Molecular Science (BNLMS), College of Chemistry and the Peking University, Beijing 100871, China.

${ }^{2}$ Lab of Computational Chemistry and Drug Design, State Key Laboratory of Chemical Oncogenomics, Peking University Shenzhen Graduate School, Shenzhen 518055, China.

${ }^{3}$ Shenzhen Bay Laboratory, Shenzhen 518055, China.

${ }^{4}$ Laboratory of Chemical Genomics, School of Chemical Biology and Biotechnology, Peking University Shenzhen Graduate School, Shenzhen 518055, China.

*Corresponding author. Email: zyang@pku.edu.cn; wuyd@pkusz.edu.cn; jhchen@pku.edu.cn.

One Sentence Summary: Discovery of an enzyme-like catalyst for highly exo-selective DielsAlder reaction of mono- and di-carbonyl activated dienophiles.

Abstract: Catalytic asymmetric Diels-Alder reaction is one of the most powerful reactions in organic chemistry. It is still a challenge to achieve high and general exo-selectivity. A novel bispyrrolidine diboronate compound has been derived from the reaction of two molecules of oxazaborolidine with one molecule of water. Upon activation, it effectively catalyzes $(0.4-5 \mathrm{~mol} \%$ loading) the Diels-Alder reaction between a variety of mono- and di-carbonyl activated dienophiles and dienes with better than 20:1 regioselectivity, up to >99:1 enantioselectivity, and better than 20:1 exo/endo selectivity. Mechanistic studies show a remarkable nonlinearity in enantioselectivity, and a second-order kinetics with respect to the catalyst concentration. A model is proposed to rationalize the above observations. The reaction is expected to find wide applications in organic synthesis.

\section{Main Text:}

The Diels-Alder reaction is one of the most useful and powerful reactions in organic chemistry (1), as exemplified by its broad applications in natural product synthesis (2). The power of this reaction is also acknowledged by industrial chemists because of the rapid and atom economical construction of complex structures with minimal waste generation (3). As up to four new stereocenters can be generated in a single 
step, tremendous efforts have been diverted to the development of asymmetric Diels-Alder reactions (4), including enzyme-catalyzed ones (5). Notable enantioselective catalysts include amine catalyst (1), oxazaborolidine (2)/acid catalyst, and the more recent silylium-carbanion pair chiral catalyst (3) as shown in Fig. 1a (6-10). These catalysts have been widely applied to Diels-Alder reactions of mono-activated dienophiles with a variety of dienes with high regio-, enantio-, and endo-selectivities. It is, however, challenging to achieve general exo-selectivity, although there have been sporadic successes employing sterically bulky dienes or dienophiles (11-13), as well as by using antibodies and modified enzymes as catalysts $(14,15)$.

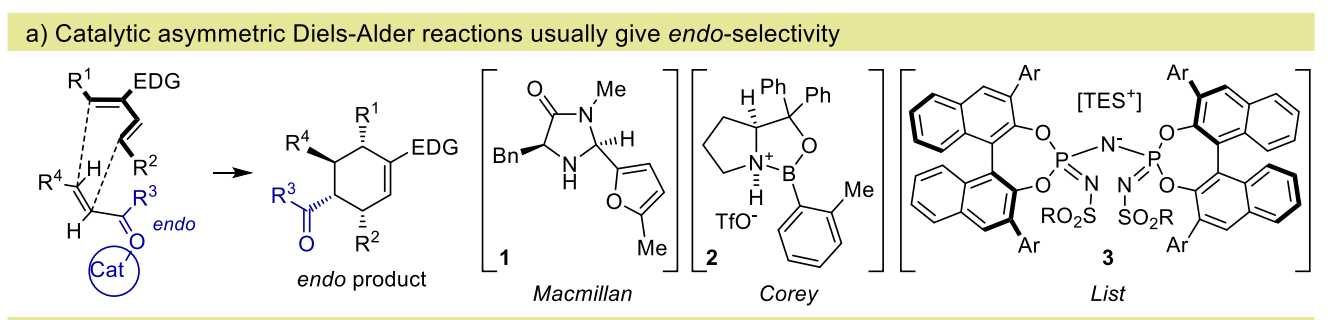

b) Examples of strategy for the diactivated dienophile-involved Diels-Alder reactions

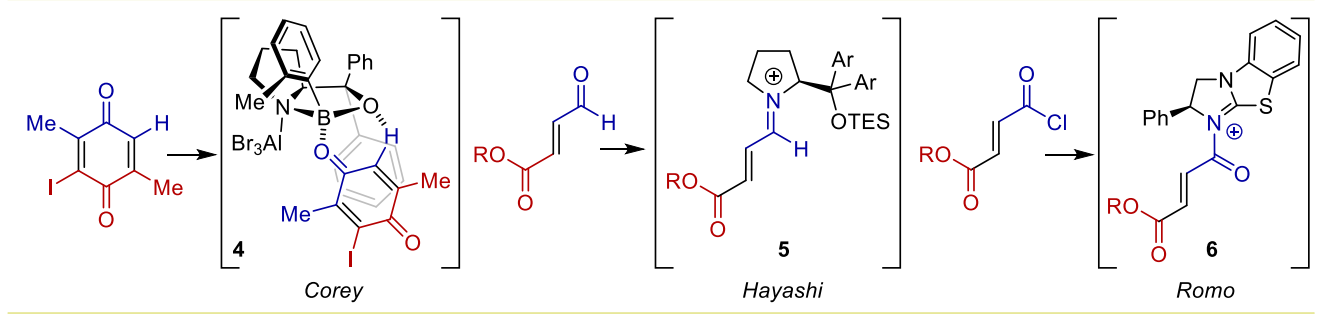

c) This work: activated BPDB as a general catalyst for Diels-Alder reactions with mono- and diactivated dienophiles

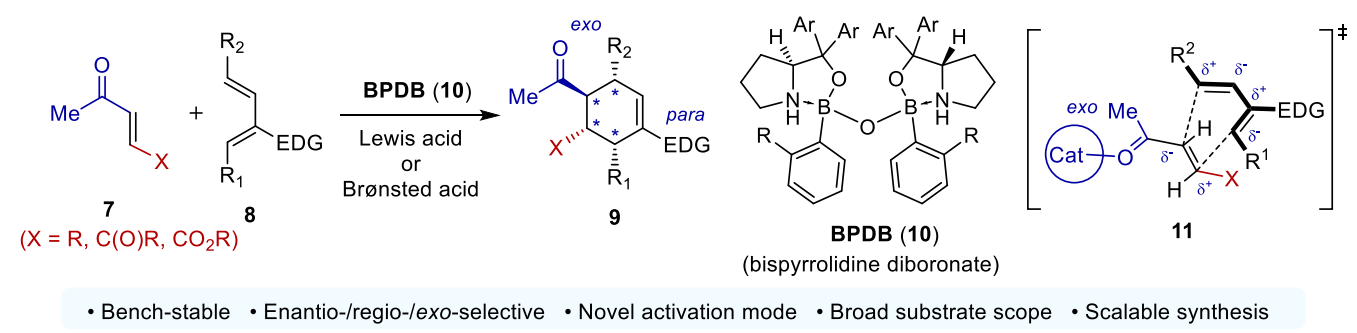

Fig. 1. Catalytic Asymmetric Diels-Alder Reactions.

Catalytic asymmetric Diels-Alder reaction of diactivated dienophiles is important, but it is generally considered to be more challenging $(16,17)$, because the reaction often produces a mixture of regioisomers. Most previous methodologies solve this problem by covalently recognizing one of the electron-withdrawing groups on the 1,2-diactivated dienophile, such as aldehydes $(18,19)$ or acyl halides (20) (Fig. 1b), which require a large difference between the two electron-withdrawing groups. Other workarounds, such as introducing a chiral fragment on the diene partner (21-23) or using symmetric dienophiles like maleimide, benzoquinone derivatives, and trans-fumarates (Fig. 1b) are also limited to specific types of substrates (24). Recently, we introduced electron-withdrawing groups onto Corey's catalyst 2 and found that such catalysts could catalyze the DielsAlder reaction of (E)-4-oxopent-2-enoates in a regioselective and enantioselective manner (25). 
Herein, we report a catalyst BPDB (10, Fig. 1c), which upon activation by a Brønsted acid or a Lewis acid, catalyzes the Diels-Alder reaction of both mono-activated and diactivated dienophiles (7) to give excellent regio-, enantio-, and more importantly, exo-selectivity. As illustrated by transition state model $\mathbf{1 1}$ (Fig. 1c), it is the acetyl group that is activated by the catalyst. This group is para to the electron-donating group of the diene, and is exo in the transition state to give product 9.

Preparation of BPDB (10). In the process of preparing CBS-12, we found that the catalyst could be converted into BPDB (10) by the treatment with $\mathrm{H}_{2} \mathrm{O}\left(0.5\right.$ eq.) in THF at $60^{\circ} \mathrm{C}$ for $30 \mathrm{~min}$. BPDB (10) was obtained in $82 \%$ isolated yield after recrystallization, and the reaction could be carried out in 20 gram-scale (Fig. 2a). BPDB (10) is a complex of bispyrrolidine diboronate, and its structure has been confirmed by X-ray crystallographic analysis (Fig. 2b). Unlike its precursor oxazaborolidine 12, BPDB (10) is air and moisture stable, and can be stored at room temperature for six months without noticeable degradation.

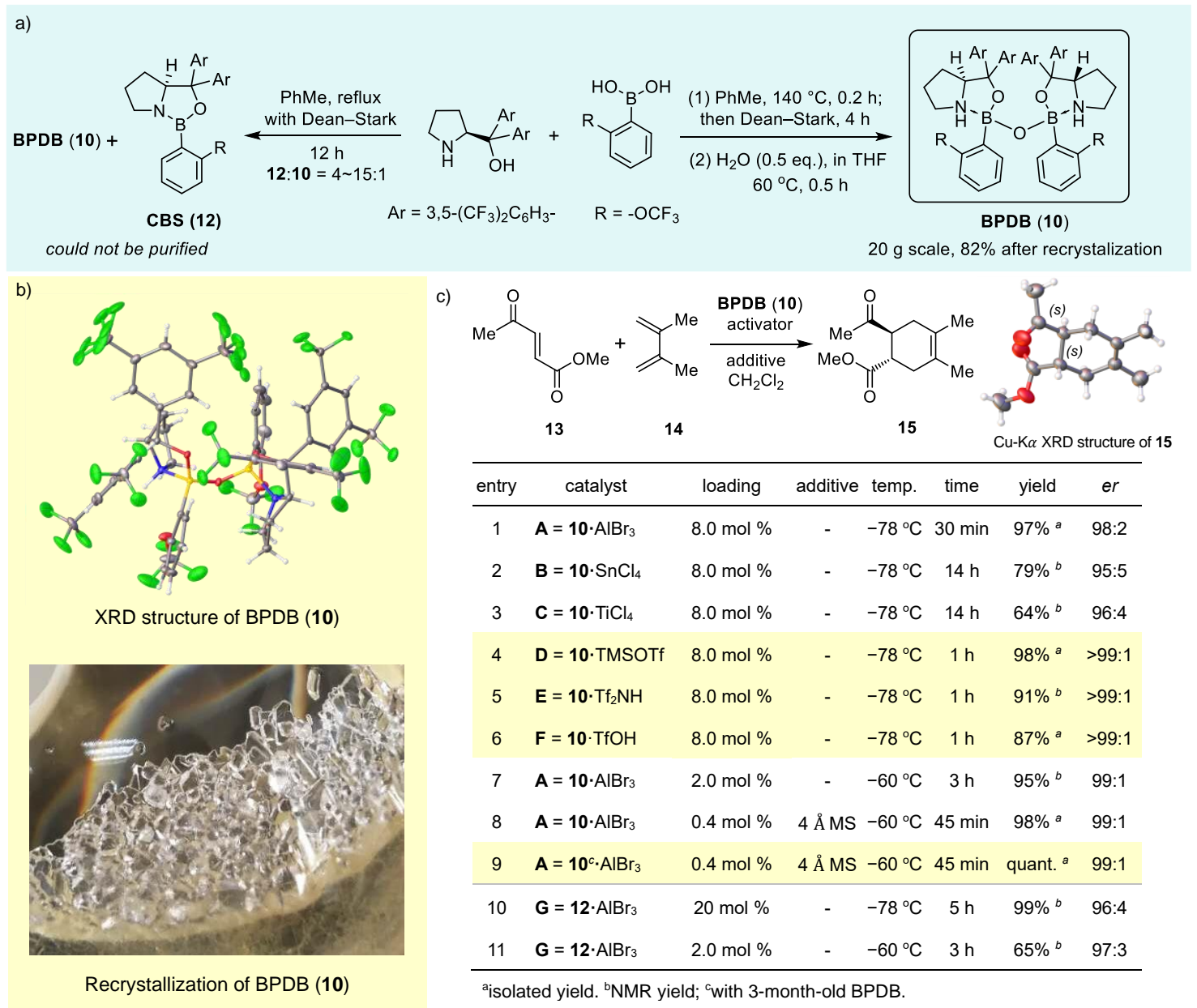

Fig. 2. Discovery of BPDB (10). (a) Preparation of BPDB; (b) Single-crystal X-ray diffraction analysis-derived structure (ORTEP representation at 50\% probability) and an image for the recrystallization of BPDB (10); (c) BPDB catalyzed DielsAlder reaction between dienophile $\mathbf{1 3}$ and diene $\mathbf{1 4 .}$ 
The BPDB-catalyzed asymmetric Diels-Alder reaction (10) was first evaluated with methyl (E)-4-oxopent2-enoate (13) and 2,3-dimethylbuta-1,3-diene (14). BPDB (10) was activated by a series of Lewis acids or Brønsted acids in a 1:1 ratio. As shown in Fig. 2c, when catalyst loading is $8 \mathrm{~mol} \%$, in $\mathrm{CH}_{2} \mathrm{Cl}_{2}$ at $-78^{\circ} \mathrm{C}$, catalyst A appears to be most effective in completing the reaction (97\% yield) in 0.5 h. Catalysts $\mathbf{D}, \mathbf{E}$, and $\mathbf{F}$ (Fig. 2c) are slightly less powerful, and the reaction time has therefore been increased to $1 \mathrm{~h}$. Catalysts $\mathbf{B}$ and $\mathbf{C}$ with Lewis acids $\mathrm{SnCl}_{4}$ and $\mathrm{TiCl}_{4}$ (Fig. 2c) appear to be least effective and the reaction gives $79 \%$ and $64 \%$ yields, respectively, after $14 \mathrm{~h}$. All these reactions proceed with high enantioselectivity, better than 95:5.

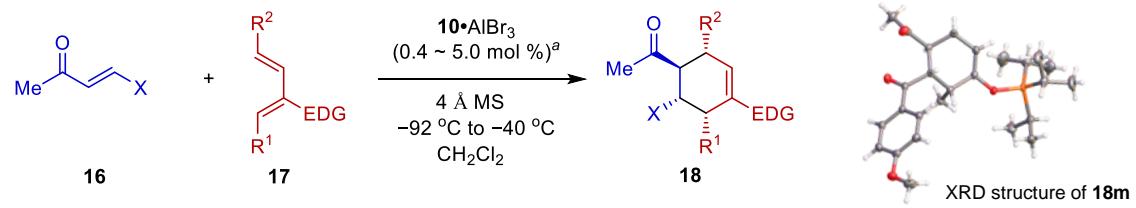

(a) reaction of ketone ester 13 with substituted dienes

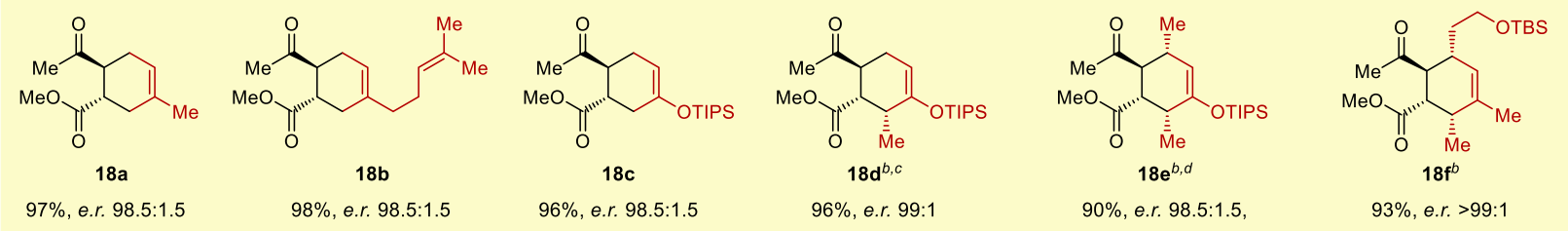

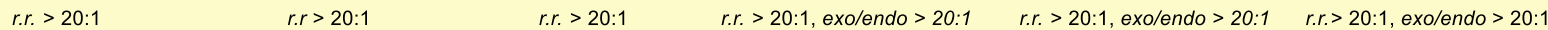

(b) reactions of 1,4-diketones with substituted dienes (18g-n), and $\alpha, \beta$-unsaturated ketones with substituted dienes (18q-s) .

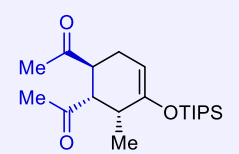

OTIPS

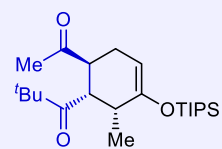

$18 \mathrm{~h}$

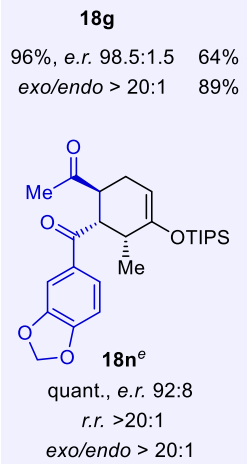

exo/endo $>20: 1$

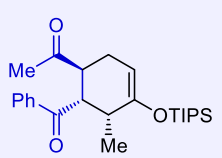

$18 \mathbf{j}^{b}$

quant., e.r. $95: 5$

r.r. $>20: 1$

exo/endo $>20$

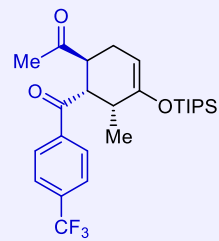

$18 \mathbf{k}^{b}$

$99 \%$, e.r. $94: 6$

r.r. $>20: 1$

exo/endo $>20$ :

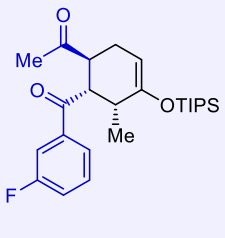

$181^{e}$

$96 \%$, e.r. $92: 8$

r.r. $>20: 1$

exo/endo $>20: 1$

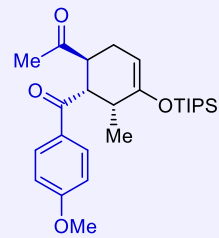

$18 \mathrm{~m}^{e}$

quant. e.r. $95: 5$

r.r. $>20: 1$

exo/endo $>20: 1$

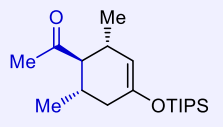

180

$90 \%$, e.r. $96: 4$

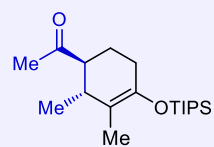

$18 \mathrm{p}^{c, f}$

$88 \%$, e.r. $96: 4$

r.r. $>20: 1$

exo/endo $>20: 1$

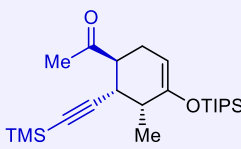

$18 \mathrm{r}^{b, c}$

$99 \%$., e.r. $>99: 1$

r.r. $>20: 1$

exo/endo $>20: 1$

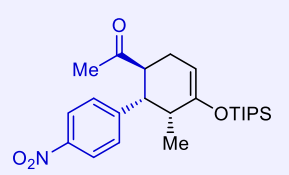

$72 \%$, e.r. $96: 4$, r.r. $>20: 1$

exo/endo > 20:1

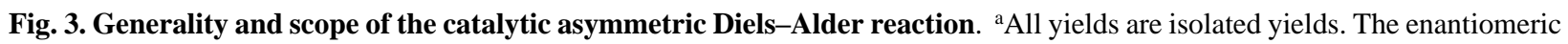
ratios of the products were determined by chiral-HPLC. The diastereo- and regioselectivities were measured by ${ }^{1} \mathrm{H}-\mathrm{NMR}$ of reaction mixtures. ${ }^{b}$ The relative configurations were determined by NMR spectroscopic analysis (NOESY, see Supporting Information). ${ }^{c}$ These reactions were carried out in the presence of TMSOTf as the activator. ${ }^{d}$ These reactions were carried out at 8 mol $\%$ catalyst loading. ${ }^{e}$ These reactions were carried out in the presence of $\mathrm{Tf}_{2} \mathrm{NH}$ as the activator at -20 to $-10{ }^{\circ} \mathrm{C}$. ${ }^{f} \mathrm{~A}$ double bond shift was observed. 
We then investigated further the more reactive catalyst $\mathbf{1 0} \cdot \mathbf{A l B r}_{\mathbf{3}}$. With a lower catalyst loading of $2.0 \mathrm{~mol} \%$, the reaction finished within 3 hours at a slightly raised temperature of $-60{ }^{\circ} \mathrm{C}$ with $95 \%$ yield and 99:1 er (Fig. 2, entry 7). Interestingly, when 4 Å MS were added, the catalyst loading could be further reduced to $0.4 \mathrm{~mol} \%$, giving a $>98 \%$ yield and 99:1 er after only 45 min (Fig. 2, entry 8), indicating that $4 \AA$ AS could act as an effective additive to significantly accelerate the reaction at low catalyst loading (26). Repeating the reaction with a 3-month-old BPDB (10) sample (Fig. 2, entry 9) gave essentially the same result as with a freshly prepared catalyst (Fig. 2, entry 8). CBS (12) shows considerably lower reactivity than BPDB (10). Entry 10 (Fig. 2) shows that CBS (12) requires higher catalyst loading (20 mol \%) and longer reaction time (5 h) for a complete conversion, giving a 99\% yield and 96:4 er. For a direct comparison with Fig. 2, Entry 7, the reaction with 2 mol \% CBS (12) loading gave only $65 \%$ yield after $3 \mathrm{~h}$ at $-60{ }^{\circ} \mathrm{C}$. The crystal structure of product 15 was determined with $\mathrm{Cu}-\mathrm{K} \alpha \mathrm{X}$-ray diffraction. It confirmed an $(S, S)$-configuration for the two generated chiral centers (Fig. 2c).

The generality and scope of this catalytic asymmetric reaction were then explored (Fig. 3). We first tested the reaction of ketone ester $\mathbf{1 3}$ with six electron-rich dienes. As shown in Fig. 3a, under optimal reaction conditions, 18a-f were obtained in good to excellent yields, along with excellent enantioselectivities. Better than 20:1 regioselectivities were also obtained for all six reactions. The major regioisomer has the acetyl group para to the electron-donating group at the $\mathrm{C} 2$ position of the dienes. For products 18d, 18e, and 18f, excellent exo-selectivities were obtained. The acetyl group is trans to the alkyl group(s) at the $\mathrm{C} 1$ or $\mathrm{C} 4$ of the dienes.

Next, the reactions of eight trans-1,4-diketone dienophiles with 1-methyl-2-triisopropylsiloxy-butadiene were investigated. The products $\mathbf{1 8} \mathbf{g}-\mathbf{n}$ are shown in Fig. 3b. All these reactions gave excellent regioselectivity with the acetyl group para to the OTIPS group as the major isomer. The regioselectivity $(r r)$ is better than 20:1 except for $\mathbf{1 8 h}$. The regioselectivity for $\mathbf{1 8 h}$ is somewhat reduced, 5:1 and $10: 1$ under $-85{ }^{\circ} \mathrm{C}$ and $-92{ }^{\circ} \mathrm{C}$, respectively. This is still quite remarkable, because it is often considered difficult to distinguish between methyl and ethyl substituents. All these reactions have excellent diastereoselectivity. Again, the acetyl group is trans to the methyl group of the diene, and exo/endo-selectivity is better than 20:1. Higher than 92:8 enantioselectivities were also obtained for these reactions.

The reactions of several mono-activated $\alpha, \beta$-unsaturated methyl ketones with electron-rich dienes were also tested. The products $\mathbf{1 8 p}-\mathbf{s}$ are shown in Fig. 3b. Again, excellent regio-, enantio-, and exo-selectivities were obtained, indicating that catalyst $\mathbf{1 0}$ can be broadly applied to the asymmetric catalysis of Diels-Alder reactions.

Mechanistic Understanding of the Asymmetric Catalysis of 10. Nonlinear effect has been very helpful in illustrating the form of catalyst in asymmetric catalysis (27). As shown in Fig. 4a, catalyst BPDB· $\mathrm{AlBr}_{3}$ exhibits a very strong positive nonlinear effect in catalyzing the reaction of $\mathbf{1 3}$ with $\mathbf{1 4}$ (Fig. 4a). A product ee of 80\% can be achieved even with a catalyst $e e$ of about $20 \%$. The nonlinearity could be fitted to a model of dimerization. 
(28) This suggests that BPDB may form dimers (or oligomers). In the crystal structure of BPDB·TfOH (19, Fig. $4 \mathrm{c}), \mathrm{BPDB}-\mathrm{H}^{+}$molecules assemble with $\mathrm{TfO}^{-}$to form a one-dimensional chain connected via hydrogen bonding (Fig. S6). We were also able to obtain crystal structures of hetero $(R, R)-(S, S)$ BPDB dimer in various solvents (Fig. S7). The dimerization constant between the hetero $(R, R)-(S, S) \mathrm{BPDB} \cdot \mathrm{AlBr}_{3}$ dimer is about 300 times that of homo $(R, R)-(R, R)$ or $(S, S)-(S, S)$ BPDB· $\mathrm{AlBr}_{3} \operatorname{dimer}\left(\mathrm{K}^{\prime} / \mathrm{K}=290\right)$. On the other hand, the homodimer is more reactive than the heterodimer by about 50 times $\left(k^{\prime} / k=0.020\right)$. Similar results were also obtained by using BPDB $\cdot \mathrm{Tf}_{2} \mathrm{NH}$ and BPDB·TfOH catalysts (Fig. S2). Interestingly, the reaction involving CBS (12, Fig. 2a) also shows a weak nonlinear effect (Fig. 4a, red line). We tentatively attribute this to the formation of a small amount of BPDB in the synthesis of CBS (see Fig. 2a).

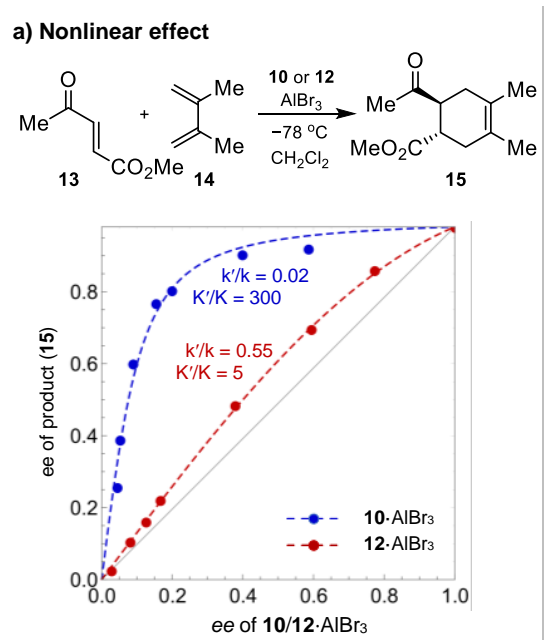

b) Kinetic study
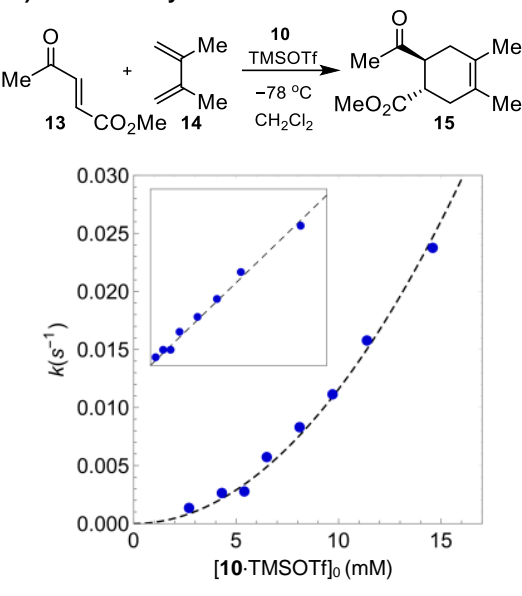

c) Single-crystal structure of BPDB·TfOH

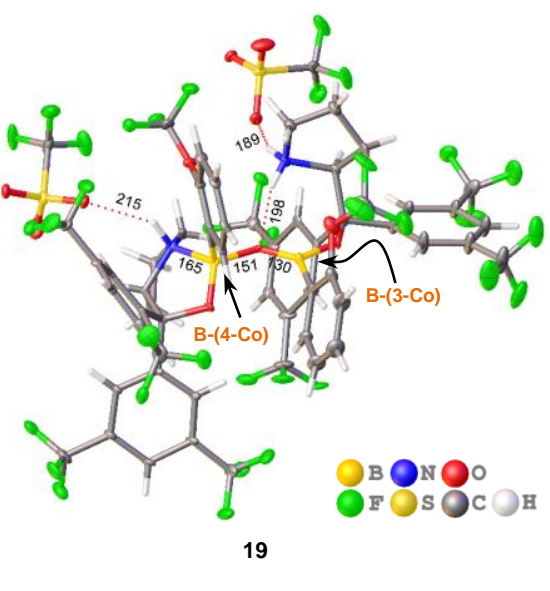

d) Proposed model
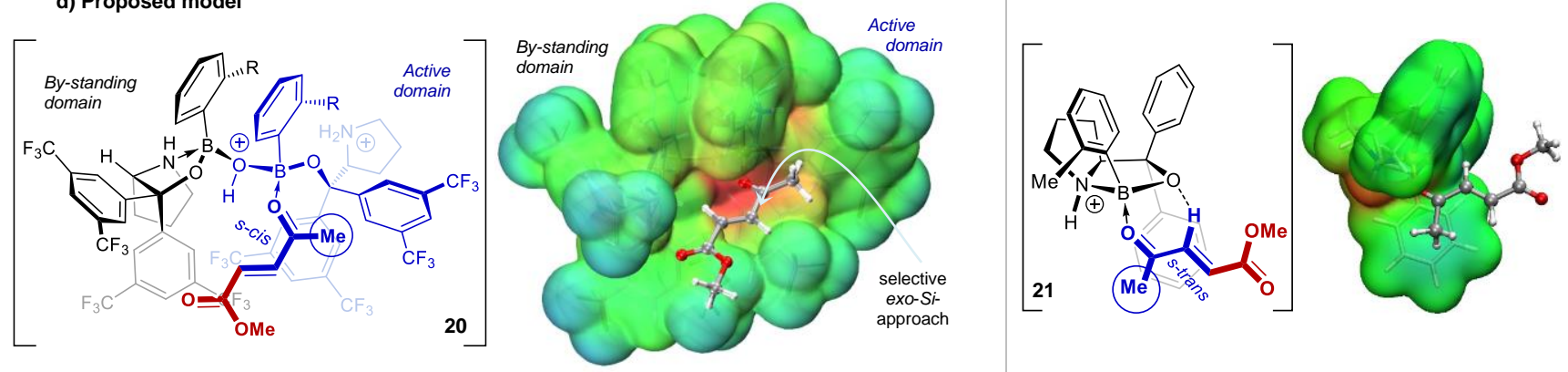

Fig. 4. Mechanistic study. (a) Nonlinear effect in the catalysis with BPDB (10)·AlBr3, blue line, and CBS (12)·AlBr 3 , red line. $\mathrm{K}^{\prime}$ and $\mathrm{K}$ are assumed $(R, R)-(S, S)$ and $(R, R)-(R, R)$ or $(S, S)-(S, S)$ dimerization constants, respectively; $\mathrm{k}^{\prime}$ and $\mathrm{k}$ are rate constant of catalysis by the $(R, R)-(S, S)$ dimer and $(R, R)-(R, R)$ or $(S, S)-(S, S)$ dimer, respectively. (b) Kinetic experiment that shows the reaction is second order with respect to the concentration of BPDB-TMSOTf. (c) Single-crystal X-ray diffraction analysis-derived structure of BPDB-TfOH (ORTEP representation at 10\% probability), which shows that one of the B-N bonds is broken (bond length labeled in $\mathrm{pm}$ ). (d) Optimized structure of the proposed active complex and model of steric control. The geometry was optimized at PBE0/def2-SV(P) level. The binding pocket is visualized by color mapping electrostatic potential (color gradient 0.04 a.u. - blue to 0.25 a.u. - red) to the isosurface (0.002 a.u.) of electron density. 
A kinetic study using in situ infrared spectroscopy was carried out with the reaction shown in Fig. 4b. Under different catalyst loadings (below $25 \mathrm{~mol} \%$ ), the fitted kinetic constant shows a second-order relationship with respect to the BPDB.TMSOTf concentration (Fig. 4b), suggesting that indeed a dimer of BPDB·TMSOTf is the active catalyst.

The single-crystal structure of BPDB-TfOH complex (19) was also obtained. As shown in Fig. 4c, one of the $\mathrm{B}-\mathrm{N}$ bonds dissociates. The freed $\mathrm{N}$-atom is protonated by the acid, and it forms a 7 -membered-ring intramolecular $\mathrm{N}-\mathrm{H} . . . \mathrm{O}$ hydrogen-bond (H-bond) with the bridging O-atom. Two $\mathrm{TfO}^{-}$groups form intermolecular $\mathrm{H}$-bonds with the adjacent $\mathrm{BPDB}-\mathrm{H}^{+}$to form a 1D super-molecular chain structure (Fig. S6). Thus, each activated BPDB possesses one of the B-atoms in tri-coordination, B(3-Co), while the other is in tetracoordination, B(4-Co). In supporting of this finding, a reaction by BPDB· $\mathrm{Tf}_{2} \mathrm{NH}$ was monitored by ${ }^{11} \mathrm{~B}-\mathrm{NMR}$ spectroscopy. Two sets of signals at $7 \mathrm{ppm}$ and $32 \mathrm{ppm}$, respectively, were observed (Fig. S1).

In the crystal structure of BPDB·TfOH complex (19), each BPDB is mono-protonated. The O-B(4-Co) and $\mathrm{O}-\mathrm{B}(3-\mathrm{Co})$ bond lengths are $151 \mathrm{pm}$ and $130 \mathrm{pm}$, respectively, indicating a significant double bond character in the $\mathrm{O}-\mathrm{B}(3-\mathrm{Co})$ bond, making the $\mathrm{B}(3-\mathrm{Co})$ site a weak Lewis acid. Indeed, calculations gave a significantly positive binding free energy for the binding of ketoester $\mathbf{1 3}$ to the $\mathrm{B}(3-\mathrm{Co})$ center (Fig. S9), indicating that it is not catalytically active.

Thus, a second acid is necessary to convert the B center to be catalytically active. This can be achieved by a proton shuffle in the BPDB-TfOH dimer. We found a structure with a second protonation on the bridge-O to be reasonable (Fig. S10). The proton transfer to the bridge-O atom breaks the 7-member-ring H-bond. The protonation of the bridge- $\mathrm{O}$ atom makes the $\mathrm{B}(3-\mathrm{Co})$ much stronger Lewis acid. Calculations show that it binds the substrate strongly (Fig. S11). This model rationalizes the observed second-order kinetics of the catalyst (Fig. 4b).

With this active catalyst, an extensive conformational search was carried out for the binding of ketone ester substrate 13 (Fig. S11). As shown in Fig. 4d, the most stable complex is structure 20. The acetyl group binds to the $\mathrm{B}(3-\mathrm{Co})$ much stronger than the ester group. It binds to the $\mathrm{B}(3-\mathrm{Co})$ in such a way that the methyl group is adjacent to the non-bridging $\mathrm{O}$ atom, and the $(\mathrm{B}) \ldots \mathrm{O}=\mathrm{C}-\mathrm{C}=\mathrm{C}$ favors an s-cis conformation over an strans conformation. These conformational features are due to the special cavity of the catalyst. The preferred acetyl binding explains the observed regioselectivity. For enantioselectivity, the backside of the dienophile is blocked by one of the Ar groups, so diene has to approach from the Si-face of the coordinating carbonyl group, resulting in the $(S, S)$ enantioselectivity for the product formation. Exo addition of the diene is more favorable than endo addition due to the steric effect caused by the by-standing boronate (29), as indicated by the stereo view of $\mathbf{2 0}$ in Fig. $4 \mathrm{~d}$.

For CBS catalysis, it is well established that the substrate binds in a way that the $\mathrm{C}=\mathrm{C}$ bond is close to the catalyst $\mathrm{O}(\mathbf{2 1})$ to facilitate a $\mathrm{C}-\mathrm{H} \ldots \mathrm{O}$ interaction $(7,30)$. The $(\mathrm{B}) \ldots \mathrm{O}=\mathrm{C}-\mathrm{C}=\mathrm{C}$ has to be s-trans. This has a significant consequence on regioselectivity. It becomes more difficult to distinguish between acetyl binding and ester (or another carbonyl group) binding, resulting in low regioselectivity. Because the substrate is in an s-trans 
conformation, the $\mathrm{C}=\mathrm{C}$ bond is extended far enough from the catalyst, so that steric effect is not significant. Therefore, endo selectivity can be achieved with the intrinsic endo preference of a transition state.

In summary, BPDB (10) is easy to prepare from CBS (12). It is air and moisture-stable. Upon activation, it can catalyze exo-selective Diels-Alder reactions of a range of diactivated and mono-activated dienophiles with high regio-, and enantio-selectivity. The exo-selectivity opens a new window for the asymmetric catalytic DielsAlder reaction. The two carbonyl groups in the product can be readily converted into useful molecular structures that are valuable in natural product synthesis $(31,32)$. The catalyst is expected to find applications in other types of catalytic asymmetric reactions. The preparation of BPDB from CBS and water may also provide a new strategy for the development of new catalysts. This catalyst, indeed, may possess some property of a DielsAlderase, a topic currently actively pursued $(33,34)$.

\section{References and Notes:}

1. O. Diels, K. Alder, Synthesen in der hydroaromatischen Reihe. Justus Liebigs Ann. Chem. 460, 98-122 (1928).

2. K. C. Nicolaou, S. A. Snyder, T. Montagnon, G. Vassilikogiannakis, The Diels-Alder Reaction in Total Synthesis. Angew. Chem. Int. Ed. 41, 1668-1698 (2002).

3. J. A. Funel, S. Abele, Industrial Applications of the Diels-Alder Reaction. Angew. Chem., Int. Ed.52, 3822-3863 (2013).

4. E. J. Corey, Catalytic Enantioselective Diels-Alder Reactions: Methods, Mechanistic Fundamentals, Pathways, and Applications. Angew. Chem. Int. Ed. 41, 1650-1667 (2002).

5. J. B. Siegel, A. Zanghellini, H. M. Lovick, G. Kiss, A. R. Lambert, J. L. St.Clair, J. L. Gallaher, D. Hilvert, M. H. Gelb, B. L. Stoddard, K. N. Houk, F. E. Michael, D. Baker, Computational Design of an Enzyme Catalyst for a Stereoselective Bimolecular Diels-Alder Reaction. Nature 329, 309-313 (2010).

6. A. B. Northrup, D. W. C. MacMillan, The First General Enantioselective Catalytic Diels-Alder Reaction with Simple $\alpha, \beta$-Unsaturated Ketones. J. Am. Chem. Soc. 124, 2458-2460 (2002).

7. E. J. Corey, T. Shibata, T. W. Lee, Asymmetric Diels-Alder Reactions Catalyzed by a Triflic Acid Activated Chiral Oxazaborolidine. J. Am. Chem. Soc. 124, 3808-3809 (2002).

8. E. J. Corey, Enantioselective Catalysis Based on Cationic Oxazaborolidines. Angew. Chem. Int. Ed. 48, 2100-2117 (2009).

9. T. Gatzenmeier, M. v. Gemmeren, Y. W. Xie, D. Höfler, M. Leutzsch, B. List, Asymmetric Lewis Acid Organocatalysis of the Diels-Alder Reaction by a Silylated C-H Acid. Science 351, 949-952 (2016).

10. T. Gatzenmeier, M. Turberg, D. Yepes, Y. W. Xie, F. Neese, G. Bistoni, B. List, Scalable and Highly Diastereo- and Enantioselective Catalytic Diels-Alder Reaction of $\alpha, \beta$-Unsaturated Methyl Esters. J. Am. Chem. Soc. 140, 12671-12676 (2018). 
11. Y. H. Lam, P. H.-Y. Cheong, J. M. B. Mata, S. J. Stanway, V. Gouverneur, K. N. Houk, Diels-Alder Exo Selectivity in Terminal-Substituted Dienes and Dienophiles: Experimental Discoveries and Computational Explanations. J. Am. Chem. Soc. 131, 1947-1957 (2009).

12. Z. J. Liu, X. L. Lin, N. Yang, Z. S. Su, C. W. Hu, P. H. Xiao, Y. Y. He, Z. L. Song, Unique Steric Effect of Geminal Bis(silane) To Control the High Exoselectivity in Intermolecular Diels-Alder Reaction. J. Am. Chem. Soc. 138, 1877-1883 (2016).

13. K. Ishihara, K. Nakano, Design of an Organocatalyst for the Enantioselective Diels-Alder Reaction with $\alpha$-Acyloxyacroleins. J. Am. Chem. Soc. 127, 10504-10505 (2005).

14. M. Sato, S. Kishimoto, M. Yokoyama, C. S. Jamieson, K. Narita, N. Maeda, K. Hara, H. Hashimoto, Y. Tsunematsu, K. N. Houk, Y. Tang, K. Watanabe, Catalytic Mechanism and endo-to-exo Selectivity Reversion of an Octalin-Forming Natural Diels-Alderase. Nat. Catal. 4, 223-232 (2021).

15. A. Heine, E. A. Stura, J. T. Yli-Kauhaluoma, C. S. Gao, Q. L. Deng, B. R. Beno, K. N. Houk, K. D. Janda, I. A. Wilson, An Antibody exo Diels-Alderase Inhibitor Complex at 1.95 Angstrom Resolution. Science, 279, 1934-1940 (1998).

16. S. Mukherjee and E. J. Corey, [4+2] Cycloaddition Reactions Catalyzed by a Chiral Oxazaborolidinium Cation. Reaction Rates and Diastereo-, Regio-, and Enantioselectivity Depend on Whether Both Bonds Are Formed Simultaneously. Org. Lett. 12, 1024-1027 (2010).

17. K. Maruoka, S. Saito, H. Yamamoto, Discrimination of Two Different Ester Carbonyls with Methylaluminum Bis(2,6-di-tert-butyl-4-methylphenoxide). Application to the Regiocontrolled and Stereocontrolled Diels-Alder Reaction of Unsymmetrical Fumarates. J. Am. Chem. Soc. 114, 10891090 (1992).

18. H. Gotoh, Y. Hayashi, Diarylprolinol Silyl Ether as Catalyst of an exo-Selective, Enantioselective Diels-Alder Reaction. Org. Lett. 9, 2859-2862 (2007).

19. L. You, X.-T. Liang, L.-M. Xu, Y.-F. Wang, J.-J. Zhang, Q. Su, Y.-H. Li, B. Zhang, S.-L. Yang, J.-H. Chen, Z. Yang, Asymmetric Total Synthesis of Propindilactone G. J. Am. Chem. Soc. 137, 1012010123 (2015).

20. M. E. Abbasov, B. M. Hudson, D. J. Tantillo, D. Romo, Acylammonium Salts as Dienophiles in DielsAlder/Lactonization Organocascades. J. Am. Chem. Soc. 136, 4492-4495 (2014).

21. Z.-J. Jia, H. Jiang, J.-L. Li, B. Gschwend, Q.-Z. Li, X. Yin, J. Grouleff, Y.-C. Chen, K. A. Jørgensen, Trienamines in Asymmetric Organocatalysis: Diels-Alder and Tandem Reactions. J. Am. Chem. Soc. 133, 5053-5061 (2011).

22. Y. Liu, M. Nappi, E. C. Escudero-Adan, P. Melchiorre, Multicatalytic Asymmetric Synthesis of Complex Tetrahydrocarbazoles Via a Diels-Alder/Benzoin Reaction Sequence. Org. Lett. 14, 13101313 (2012).

23. Y. Wang, H. Li, Y.-Q. Wang, Y. Liu, B. M. Foxman, L. Deng, Asymmetric Diels-Alder Reactions of 2-Pyrones with a Bifunctional Organic Catalyst. J. Am. Chem. Soc. 129, 6364-6365 (2007). 
24. D. Liu, E. Canales, E. J. Corey, Chiral Oxazaborolidine-Aluminum Bromide Complexes Are Unusually Powerful and Effective Catalysts for Enantioselective Diels-Alder Reactions. J. Am. Chem. Soc. 129, 1498-1499 (2007).

25. S.-L. Zhang, Y. Lu, Y.-H. Li, K.-Y. Wang, J.-H. Chen, Z. Yang, Catalytic and Enantioselective DielsAlder Reactions of (E)-4-Oxopent-2-Enoates. Org. Lett. 19, 3986-3989 (2017).

26. K. Mikami, M. Terada, T. Nakai, Catalytic Asymmetric Glyoxylate-Ene Reaction: A Practical Access to $\alpha$-Hydroxy Esters in High Enantiomeric Purities. J. Am. Chem. Soc. 112, 3949-3954 (1990).

27. H. B. Kagan, T. O. Luukas, In Comprehensive Asymmetric Catalysis; E. N., Jacobsen, A. Pfaltz, H. Yamamoto, Eds.; Springer-Verlag: Berlin, 1999; Vol. I, Chapter 4.1, 101-118.

28. C. Puchot, O, Samuel, E. Dunach, S. Zhao, C. Agami, H. B. Kagan, Nonlinear Effects in Asymmetric Synthesis. Examples in Asymmetric Oxidations and Aldolization Reactions. J. Am. Chem. Soc. 108, 2353-2357 (1986).

29. J. Qi, W. R. Roush, Synthesis of Precursors of the Agalacto (Exo) Fragment of the Quartromicins via an Auxiliary-Controlled Exo-Selective Diels-Alder Reaction. Org. Lett. 8, 2795-2798 (2006).

30. M. N. Paddon-Row, C. D. Anderson, K. N. Houk, Computational Evaluation of Enaqntioselective Diels-Alder Reactions Mediated by Corey's Cationic Oxazaborolidine Catalysts. J. Org. Chem. 74, 861-868 (2009).

31. D.-D. Liu, T.-W. Sun, K.-Y. Wang, Y. Lu, S.-L. Zhang, Y.-H. Li, Y.-L. Jiang, J.-H. Chen, Z. Yang, Asymmetric Total Synthesis of Lancifodilactone G Acetate. J. Am. Chem. Soc. 139, 5732-5735 (2017).

32. Y.-L. Jiang, H.-X. Yu, Y. Li, P. Qu, Y.-X. Han, J.-H. Chen, Z. Yang, Asymmetric Total Synthesis of Pre-Schisanartanin C. J. Am. Chem. Soc. 142, 573-580 (2020).

33. L. Gao, C. Su, X.-X. Du, R.-S. Wang, S.-M. Chen, Y. Zhou, C.-W. Liu, X.-J. Liu, R.-Z. Tian, L.-Y. Zhang, K.-B. Xie, S. Chen, Q.-Q. Guo, L.-P. Guo, Y. Hano, M. Shimazaki, A. Minami, H. Oikawa, N. Huang, K. N. Houk, L.-Q. Huang, J.-G. Dai, X.-G. Lei, FAD-Dependent Enzyme-Catalysed Intermolecular [4+2] Cycloaddition in Natural Product Biosynthesis. Nat. Chem. 12, 620-628 (2020)

34. M. J. Byrne, N. R. Lees, L. C. Han, M. W. Kamp, A. J. Mulholland, J. E. M. Stach, C. L. Willis, P. R. Race, The Catalytic Mechanism of a Natural Diels-Alderase Revealed in Molecular Detail. J. Am. Chem. Soc. 138, 6095-6098 (2016).

Acknowledgments: We thank Prof. Jian Pei, Dr. Rong Zhu, Dr. Jie Su, Dr. Zhang Xiu of Peking University, and Dr. Nan Zi-Ang of Xiamen University for insightful discussions. The computational studies were supported by the SZBL supercomputing center and the high-performance computing platform of PKU. Funding: Supported by the National Science Foundation of China (Grant Nos. 21772004, 21871012, 21933004, 21632002), the Key-Area Research and Development Program of Guangdong Province (2020B010188001). Author contributions: Conceptualization: Zhen Yang; Supervision: Zhen Yang, Yun-Dong Wu, Jia-Hua Chen; Experimental investigation: Yuan-He Li, Su-Lei Zhang; Computational investigation: Bo Xiao, Tian-Yu Sun, Yuan-He Li; Writing - original draft: Yuan-He Li; Writing - review \& editing: all authors. Competing interests: The authors declare no competing interests. Data and materials availability: Refined single-crystal X-ray crystallographic results for the structure of $\mathbf{1 0}$ (racemic and enantiomerically pure ones in various solvents), $\mathbf{1 5}, \mathbf{1 8 m}, 19$ are available free of charge from the 
Cambridge Crystallographic Data Centre under deposition number CCDC $2104966(\mathbf{1 0}), 2104967(( \pm)$ 10), 2104971 (15), 2104970 (18m), 2104955 (19), 2104957 (10·DCM), 2104961 (10·3(DMSO)), 2104969 (10-6(THF)). Additional discussion, experimental procedures, characterization of new compounds, and computational results are available in the supplementary materials.

\section{Supplementary Materials:}

Materials and Methods

Figures S1-S13

Tables S1-S3 